\title{
An Auto-Delegation Mechanism for Access Control Systems
}

\author{
Jason Crampton and Charles Morisset \\ Information Security Group, \\ Royal Holloway, University of London, \\ Egham, Surrey TW20 0EX, U.K. e-mail: \\ firstname.lastname@rhul.ac.uk
}

\begin{abstract}
Delegation is a widely used and widely studied mechanism in access control systems. Delegation enables an authorized entity to nominate another entity as its authorized proxy for the purposes of access control. Existing delegation mechanisms tend to rely on manual processes initiated by end-users. We believe that systems in which the set of available, authorized entities fluctuates considerably and unpredictably over time require delegation mechanisms that can respond automatically to the absence of appropriately authorized users. To address this, we propose an auto-delegation mechanism and explore the way in which such a mechanism can be used to provide (i) controlled overriding of policy-based authorization decisions (ii) a novel type of access control mechanism based on subject-object relationships.
\end{abstract}

\section{Introduction}

An access control system is a mechanism that grants or denies requests made by active entities, the subjects, to access some passive entities, the objects. Such a mechanism usually consists of two parts, as shown in Fig. 1: the Policy Decision Point (PDP), which analyzes a request and decides whether it should be granted or not; and the Policy Enforcement Point (PEP), to which the access request is submitted and which grants the request based on the decision of the PDP.

Conceptually, a PDP is usually implemented as a function that takes an access request and an authorization policy as inputs and returns a decision indicating whether the request is authorized by the policy. The most well-known models for the definition of a PDP - such as Bell-LaPadula [20], RBAC [12], the Chinese Wall [3] or discretionary models $[15,19]$ - consider an access from a "subject-centered" point of view: a subject is authorized to access an object if she is qualified enough and/or if granting such an access would not create an illicit information flow [11]. The notion of qualification depends on the system and the policy enforced, but usually the level of qualification of a subject is defined regardless of the other subjects. As a result, when no sufficiently qualified subject exists to access an object, this object cannot be accessed at all. The inaccessibility of certain objects, such as healthcare records or essential military intelligence, could be a severe limitation or even life-threatening in some contexts. 


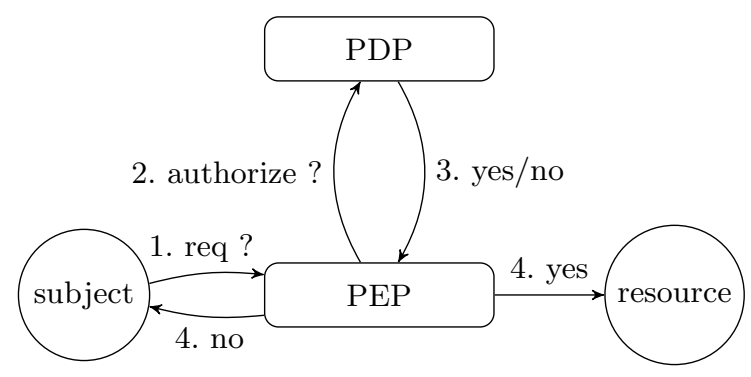

Fig. 1. Access Control System

Two different approaches exist to address this challenge. The first is to define a different authorization policy for each context, either by introducing directly the notion of context in the policy [17], or by defining specific overriding ("breakthe-glass") policies to be applied when an emergency occurs $[1,26]$. However, these policies do not take into account the existence or availability of qualified subjects; instead they extend the set of authorized accesses for the duration of then emergency, therefore possibly allowing a (normally) unauthorized subject to access an object, even though authorized subjects are available.

The second approach is to use a delegation mechanism [6]: when a subject cannot access an authorized resource, she can delegate her right to do so to another subject. ${ }^{1}$ Such an approach requires the delegation to be activated beforehand; that is, a subject must know when she will be unavailable in order to delegate her rights at this time. However, in some situations, the unavailability of a subject can be unexpected, for instance a subject getting injured or killed on a battlefield. In such a situation, how should the rights of this subject be delegated? Moreover, the delegating user must choose the delegate, and either the choice is not constrained, allowing the user to choose a delegate with little qualification, even though better qualified users are available, or the choice is constrained, thus requiring another policy. The approach presented here introduces, among other notions, such a policy.

In this paper, we present an automatic delegation mechanism (ADM), which is "object-centered": an object can be accessed by one of the most qualified subject available. This mechanism relies on the definition, for each object, of a partially ordered set of qualifications, each subject being assigned to a qualification. A notion of availability is also introduced, and the mechanism is implemented using either a standard access control monitor or a distribution scheme for cryptographic keys. The main contribution of this paper is thus to provide a mechanism which can automatically handle situations where no qualified user exists to perform (or to delegate) a time-critical task.

\footnotetext{
${ }^{1}$ We take the conventional view that a subject is a program that has been caused to execute by a human user and whose subsequent actions are controlled by that user.
} 
The rest of this paper is organized as follows: Section 2 introduces the ADM and illustrates it for an instance of the RBAC model and an instance of the BellLaPadula model. Section 3 describes different approaches to enforce an ADM. Section 4 describes the implementation of the mechanism. Section 5 concludes and discusses possibilities for future work.

\section{An Object-Centric Auto-Delegation Mechanism}

We write $\mathcal{S}=\left\{s_{1}, s_{2}, \cdots, s_{n}\right\}$ for the set of subjects, $\mathcal{O}=\left\{o_{1}, o_{2}, \cdots, o_{m}\right\}$ for the set of objects and $\mathcal{A}$ for the set of access modes (e.g. read, write, execute). An access is a tuple $(s, o, a)$, meaning that the subject $s$ accesses the object $o$ according to the access mode $a$. As we said in the introduction, an access is authorized if the subject is one of the most qualified available subject, therefore we first introduce a notion of availability and then the security policy itself based on a notion of qualification. Finally, we describe a way to build a set of qualifications for instances of the RBAC and Bell-LaPadula policies.

\subsection{Availability of Subjects}

The ADM makes decisions based on the availability of subjects. The definition of availability will be application-specific. In the context of a hospital, a physician may have authenticated to the hospital's computer systems, but may, for example, be in theater and unable to respond to any emergency that arises in relation to the care of another of her patients. In the military setting, availability may simply be whether the corresponding user is still alive.

By definition, the construction of a list of available subjects will require the run-time monitoring of the system. At its simplest, such a list might be defined by the set of users that are currently authenticated to the system.

It is likely, however, that the set of available subjects, and the meaning of "available", will be more complex. For instance, suppose we have a workflow management system (WfMS) and that there exist constraints on the execution of tasks (in the sense that if a user $u$ performs task $t$ in some instance of a workflow then $u$ cannot perform some subsequent task $t^{\prime}$ in that workflow instance). Now the WfMS will maintain some authorization policy, which determines the authorized users for each task. The WfMS could also maintain an "unavailable list" for each task which is updated whenever a preceding task in the workflow is executed that affects the set of authorized users. In the above example, the unavailable list for task $t^{\prime}$ would have $u$ added to it once $u$ executes task $t$.

Hence, there will be a need for systems that can rapidly and reliably "poll" subjects to determine their availability. Whether this polling takes place periodically, or when there is a "context shift" to an emergency mode of operation, will, again, be application-dependent.

Ignoring these application-specific considerations, we assume that it is possible to identify a set of available subjects at request evaluation time, which is a subset of all subjects and is denoted by $A v(\mathcal{S})$. 


\subsection{The ADM Policy}

Given an object $o \in \mathcal{O}$ we first define a qualification hierarchy $\left(Q(o), \leqslant_{o}\right)$. We then assume the existence of a qualification function $\lambda_{o}: \mathcal{S} \rightarrow Q(o)$, where $\lambda_{o}(s)$ denotes the qualification level of $s$, with respect to $o$. Given two subjects $s_{1}$ and $s_{2}, \lambda_{o}\left(s_{1}\right) \leqslant_{o} \lambda_{o}\left(s_{2}\right)$ means that $s_{2}$ is more qualified than $s_{1}$ to access $o$. For ease of exposition, we consider here that the qualification of a subject for an object is independent of the access mode, i.e. a subject is equally qualified to read, to write or to execute an object. A straight-forward way to take access modes into consideration is to define qualifications over permissions, where a permission is a pair (object, access-mode), as defined in the RBAC model. However, more elaborate approaches could be used to integrate the notion of access modes; we consider this to be future work. Henceforth, we will assume that the qualification of a subject for an object is the same for any access mode. Moreover, we will omit the subscript $o$ except where it is necessary to distinguish between different objects.

The relation $\leqslant$ is a partial-order, therefore two qualifications $q_{1}$ and $q_{2}$ might not be comparable, in which case there is no precedence between subjects assigned to $q_{1}$ and subjects assigned to $q_{2}$. When two subjects have the same qualification with respect to an object, they are said to be equally qualified to access the object.

We define a function $A u t h_{a d m}$ which, given $\leqslant, A v(\mathcal{S})$, and an access request $(s, o, a)$, returns an authorization decision. Specifically,

$$
\begin{aligned}
& \text { Auth } \text { adm }(\leqslant, A v(\mathcal{S}),(s, o, a)) \\
& = \begin{cases}\text { deny } & \text { if there exists } s^{\prime} \in A v(\mathcal{S}) \text { such that } \lambda(s)<\lambda\left(s^{\prime}\right), \\
\text { allow } & \text { otherwise. }\end{cases}
\end{aligned}
$$

In other words, a request by $s$ to access $o$ is allowed if $s$ is one of the most qualified of the available subjects (and denied otherwise).

For instance, consider a UNIX-like system where the subjects are the processes, and the objects are the files. In the following, we say that $s$ is a child of $s^{\prime}$ if $s^{\prime}$ created $s$, and in this case we call $s^{\prime}$ the parent of $s$, denoted as $s^{\prime}=\operatorname{parent}(s)$. We define the notion of sub-process inductively: $s$ is a sub-process of $s^{\prime}$ if, and only if, $s$ is a child of $s^{\prime}$ or if parent(s) is a sub-process of $s^{\prime}$, and in this case we call $s^{\prime}$ an ancestor of $s$.

For each object $o$, we first assume a set $S_{o} \subseteq \mathcal{S}$ of subjects corresponding to the most qualified subjects for $o$. For instance, a process belongs to $S_{o}$ if, and only if, it belongs to the user owning the file, and it does not have any ancestor belonging to this user. Note that the owner of a process might be different than the owner of its parent, otherwise every process would be owned by the root.

Given an object $o$ and a set of subject $\mathcal{S}=\left\{s_{1}, s_{2}, \cdots, s_{n}\right\}$, we define $Q(o)$ as $\left\{q_{1}, q_{2}, \cdots, q_{n}\right\}$, such that $\lambda\left(s_{i}\right)=q_{i}$, for any $i>0$. The partial order over $Q(o)$ is defined as follows: given two qualifications $q_{i}$ and $q_{j}$, such that $\lambda\left(s_{i}\right)=q_{i}$ and $\lambda\left(s_{j}\right)=q_{j}, q_{i} \leqslant q_{j}$ if, and only if, $s_{i}$ is a sub-process of $s_{j}$ which is a sub-process of a subject in $S_{o}$, or $s_{i}$ is not a sub-process of any subject in $S_{o}$. Note that if the set $\mathcal{S}$ changes over time, then the set $Q(o)$ also does. 
If a process is one of the most qualified processes and becomes unavailable, all of its children can access the object. For instance, consider the situation described in Fig. 2, where the process $s_{0}$ is owned by the root and the processes $s_{1}, s_{2}, s_{3}, s_{4}$ and $s_{5}$ are owned by a user $u$, such that $s_{1}$ and $s_{4}$ are the children of $s_{0}, s_{2}$ and $s_{3}$ are children of $s_{1}$, and $s_{5}$ is a child of $s_{4}$ Given an object $o$ owned by $u$, we have $S_{o}=\left\{s_{1}, s_{4}\right\}$. If all the processes are available, only $s_{1}$ and $s_{4}$ can access the object, according to the ADM. If $s_{1}$ becomes unavailable, then $s_{2}$ and $s_{3}$ can access the object, but not $s_{5}$. On the contrary, if $s_{4}$ becomes unavailable, $s_{5}$ can access the object, but neither $s_{2}$ nor $s_{3}$. The subject $s_{0}$ becomes the most qualified subject only if every other subject is unavailable. Note that with the underlying UNIX policy, since $s_{0}$ is owned by the root, she can access $o$ anyway. The fact that she is the least qualified should not be seen as a constraint, but rather as the ability for $s_{2}, s_{3}$ and $s_{5}$ to be able to access $o$ when $s_{1}$ or $s_{4}$ are unavailable, even though $s_{0}$ is available, thus alleviating the burden on $s_{0}$.

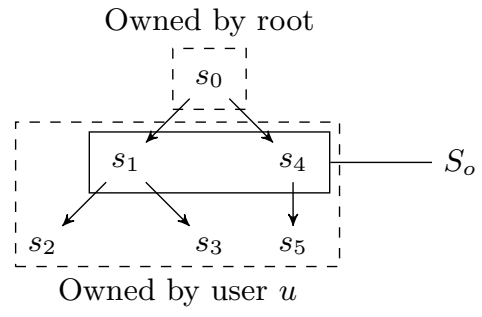

(a) Processes hierarchy

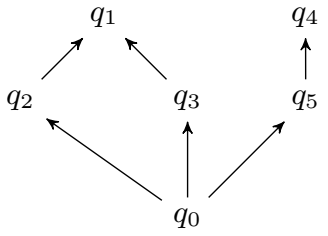

(b) $Q(o)$, o owned by $u$

Fig. 2. Qualifications for Processes

\subsection{Applications}

This section illustrates how the qualifications can be constructed for two of the most famous access control models: role-based access control (RBAC) [12] and Bell-LaPadula (BLP) [20], which have been used extensively in the medical [21, $24]$ and the military environments, respectively.

RBAC. The RBAC96 model [25], defines a set of users $\mathcal{U}$, a partially ordered set of roles $\mathcal{R}$, a set of permissions (object-access pairs) $\mathcal{P}$, and two assignment relations, $\mathrm{PA} \subseteq \mathcal{P} \times \mathcal{R}$ and $\mathrm{UA} \subseteq \mathcal{U} \times \mathcal{R}$. The assignment relations define, respectively, the permissions for which a role is authorized and the roles for which a user is authorized. A subject, more usually called a session in RBAC, is associated with a user, as usual, but it may have a subset of the roles for which that user is authorized. The partial ordering on $\mathcal{R}$ (which is usually called a role hierarchy) extends the explicit assignments defined by UA and PA to include 
implicit assignments: any user assigned to $r$ may activate any role $r^{\prime}<r$ in a session and is also authorized for the permissions of $r^{\prime}$.

We write $\operatorname{Perm}(s)$ to denote the set of permissions for which a session is authorized, where $\operatorname{Perm}(s)$ includes any permissions for which $s$ is authorized by virtue of inheritance (defined by the role hierarchy). We write $\operatorname{card}(X)$ to denote the number of elements in $X$. First, we define a distance function $\delta_{\text {rbac }}$ : $\mathcal{S} \rightarrow \mathcal{S} \rightarrow[0,1]$, where

$$
\delta_{\text {rbac }}\left(s_{1}, s_{2}\right)=1-\frac{\operatorname{card}\left(\operatorname{Perm}\left(s_{1}\right) \cap \operatorname{Perm}\left(s_{2}\right)\right)}{\operatorname{card}\left(\operatorname{Perm}\left(s_{1}\right) \cup \operatorname{Perm}\left(s_{2}\right)\right)}
$$

Hence, $\delta_{\text {rbac }}\left(s_{1}, s_{2}\right)=1$ if $\operatorname{Perm}\left(s_{1}\right) \cap \operatorname{Perm}\left(s_{2}\right)=\emptyset$ (including the degenerate case when $\left.\operatorname{Perm}\left(s_{1}\right)=\operatorname{Perm}\left(s_{2}\right)=\emptyset\right)$, and $\delta_{\text {rbac }}\left(s_{1}, s_{2}\right)=0$ if $\operatorname{Perm}\left(s_{1}\right)=$ $\operatorname{Perm}\left(s_{2}\right)$.

Given an object $o$ and the distance function $\delta_{r b a c}$, and assuming a set $S_{o}$ of subjects most qualified for $o$, we can define $\lambda_{o}: \mathcal{S} \rightarrow[0,1]$ as

$$
\lambda_{o}(s)=\min \left\{\delta_{r b a c}\left(s, s_{0}\right) \mid s_{0} \in S_{o}\right\} .
$$

The set of qualifications $Q$ is $[0,1]$, with the dual order relation: 0 is the highest qualification, since it is the closest from a subject in $S_{o}$, while 1 is the lowest qualification.

For instance, consider a system where there are four subjects $s_{1}, s_{2}, s_{3}, s_{4}$, $\operatorname{Perm}\left(s_{3}\right)=\operatorname{Perm}\left(s_{2}\right) \cup P$, and $\operatorname{Perm}\left(s_{4}\right)=\operatorname{Perm}\left(s_{2}\right) \backslash Q$, where $\operatorname{P\cap Perm}\left(s_{1}\right)=$ $\emptyset, Q \subseteq \operatorname{Perm}\left(s_{1}\right)$ and $\operatorname{card}(P)=\operatorname{card}(Q)$. Consider also an object $o$ such that $s_{1}$ is designated as the most qualified subject, in other words $S_{o}=\left\{s_{1}\right\}$. The qualifications for $o$ are then defined based on the distance from each subject to $s$. Writing $m$ and $n$ for $\operatorname{card}\left(\operatorname{Perm}\left(s_{1}\right) \cup \operatorname{Perm}\left(s_{2}\right)\right)$ and $\operatorname{card}\left(\operatorname{Perm}\left(s_{1}\right) \cap\right.$ $\left.\operatorname{Perm}\left(s_{2}\right)\right)$, respectively, and $p=\operatorname{card}(P)=\operatorname{card}(Q)$, we have

$$
\begin{aligned}
& \delta_{r b a c}\left(s_{1}, s_{2}\right)=\frac{m-n}{m} \\
& \delta_{r b a c}\left(s_{1}, s_{3}\right)=\frac{m+p-n}{m+p}=\delta_{r b a c}\left(s_{1}, s_{2}\right)+\frac{p n}{m(m+p)} \\
& \delta_{r b a c}\left(s_{1}, s_{4}\right)=\frac{m-(n-p)}{m}=\delta_{r b a c}\left(s_{1}, s_{2}\right)+\frac{p}{m}
\end{aligned}
$$

Now $n /(m+p)<1$ since $n \leqslant m$. Hence, $\delta\left(s_{1}, s_{2}\right)<\delta\left(s_{1}, s_{3}\right)<\delta\left(s_{1}, s_{4}\right)$. Then, as we discussed above, we can define $\lambda_{o}$ using the distance function $\delta_{\text {rbac }}$, as $\lambda_{o}\left(s_{4}\right)<\lambda_{o}\left(s_{3}\right)<\lambda_{o}\left(s_{2}\right)<\lambda_{o}\left(s_{1}\right)$.

We note that $\delta_{\text {rbac }}$ is not necessarily "consistent" with the role hierarchy. In particular, incomparable roles may be closer in terms of distance than junior and senior roles. For instance, a physician may be closer to a nurse, with whom she shares many permissions, than the chief medical officer, who inherits the permissions of a physician, but may have many more permissions besides.

Information Flow Policies. Reading an object can be interpreted as causing information to flow from the object (to the reader). An information flow policy 
specifies which flows of information are authorized [11]. To specify such a policy, we define:

- a partially ordered set of security labels $(L, \leqslant) ;{ }^{2}$

- a set of users $U$ and a set of objects $O$;

- a security function $f: U \cup O \rightarrow L$, where $f(x)$ denotes the security label of entity $x$.

Then a user $u$ is authorized to read $o$ if and only if $f(u) \geqslant f(o)$. Informally, information can only flow "upwards" with respect to $L$; in particular, information cannot flow from an object to a less privileged user. The standard security lattice, defined as part of the Bell-LaPadula model [20], has the form $\mathcal{C} \times 2^{\mathcal{K}}$, where $\mathcal{C}$ is a totally ordered set of security classifications (such as top secret, classified, etc.) and $\mathcal{K}$ is a set of need-to-know categories.

In the context of Bell-LaPadula (BLP), it is sufficient to define a qualification poset $Q_{l}$ for each $l \in L$ and a function $\lambda_{l}: L \rightarrow Q_{l}$. Given an object, $Q(o)$ is then defined to be $Q_{f(o)}$, and for any subject $s, \lambda(s)$ is defined to be $\lambda_{l}(f(s))$. Although it is possible to define $Q_{l}$ directly as the lattice $L$, such a definition would imply that users with the highest ranks are also the most qualified users, for any object. It would follow that as long as such users are available, they might have to take the responsibility to access many objects, which may prevent them from performing operations more appropriate to their seniority; in exceptional circumstances, we may wish instead to delegate such operations to unauthorized but adequately qualified users.

Instead of using the lattice $L$ directly, we use the intuition that, for many applications, the qualification of a security level with respect to another level will be related to the proximity of the two labels in the graph of $L$. In particular, immediate child and parent nodes should be more qualified than other, more remote nodes. Indeed, in addition to the lattice of levels of security, users are often organised as teams or task-forces, and different members of a team might have levels of security close to each other, due to the sharing of a common subset of need-to-know categories. We believe here that, in general, if a subject is unavailable to access an object she is responsible for, another subject of the same team is more qualified to access such an object, even if she is not qualified enough, rather than a subject with a very high rank. Of course, formalising exactly this property would require a precise notion of team, which does not exist in the original BLP model, and therefore, in the following construction, we take the view that the immediate child and parent nodes of a node $l$ are equally well qualified, to alleviate the burden on the parent nodes should all subjects associated with $l$ be unavailable.

Given $l \in L$, we construct the (graph of the) qualification poset $Q_{l}$ from the graph of the security lattice $(L, \leqslant)$ as follows:

- Set $l$ to be the maximal node in $Q(l)$ and define $\{l\}$ to be $N_{c}$ (the currentnode-set) and define $N_{r}$, the remaining-node-set, to be $L \backslash N_{c}$.

${ }^{2}$ In other words, $\leqslant$ is a reflexive, anti-symmetric, transitive, binary relation defined on $L$. 
- Define $N_{x}$, the next-node-set, to be all parent and child nodes of all nodes in $N_{c}$ and add an edge from $a \in N_{c}$ to $b \in N_{x}$ if $a$ is the parent or child of $b$ in $L$.

- Set $N_{x}=N_{c}$.

- Repeat the above steps until $N_{r}=\emptyset$.

An example of the lattice is given in Fig 3, for the classifications Pub (Public) and $\operatorname{Sec}$ (Secret) and the set of need-to-knows categories $\mathcal{K}=\left\{K_{1}, K_{2}\right\}$, together with the corresponding poset for the level of security $\left(S e c,\left\{K_{1}\right\}\right)$.

$$
\begin{aligned}
& l_{1}=(P u b, \emptyset) \\
& l_{2}=\left(P u b,\left\{K_{1}\right\}\right) \\
& l_{3}=\left(P u b,\left\{K_{2}\right\}\right) \\
& l_{4}=\left(P u b,\left\{K_{1}, K_{2}\right\}\right) \\
& l_{5}=(\operatorname{Sec}, \emptyset) \\
& l_{6}=\left(\operatorname{Sec},\left\{K_{1}\right\}\right) \\
& l_{7}=\left(\operatorname{Sec},\left\{K_{2}\right\}\right) \\
& l_{8}=\left(\operatorname{Sec},\left\{K_{1}, K_{2}\right\}\right)
\end{aligned}
$$

(a) Levels of security

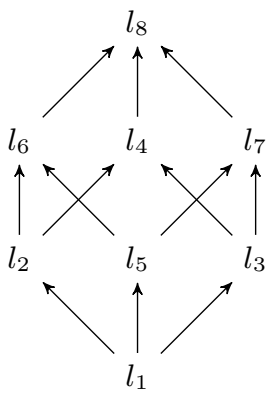

(b) Oriented graph

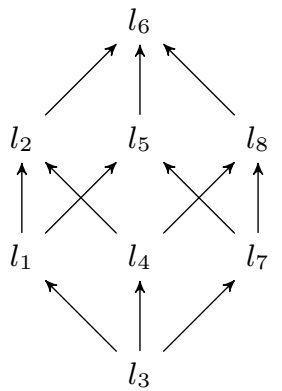

(c) Poset $Q_{l_{6}}$

Fig. 3. Information flow lattice

\section{Using the ADM}

An ADM can be deployed in two different ways within an information system: either as an exception mechanism, to supplement an existing PEP/PDP, or as a standalone mechanism.

\subsection{ADM as an Exception Mechanism}

Most access control systems are required to be conservative, or correct: the PEP should not allow requests denied by the PDP. Indeed, a request denied by the PDP means that the corresponding access violates the policy enforced by the system. However, a trivially correct PEP would deny every request, therefore making the system useless. A system can also be required to be complete: every request authorized by the PDP should be granted by the PEP. Of course, in an ideal world, every access control system should be both correct and complete, but in practice, some exceptions have to be handled.

A negative exception occurs when a decision made by the PDP to authorize a request is overridden by the PEP, which denies the request. Such exceptions 
might occur when the PEP makes use of information received from other security systems, such as an intrusion detection system.

Conversely, a PEP may override a deny response from the PDP and grant a request. Such positive exceptions are needed in mission-critical systems where human lives are at stake. The main challenge here is to allow such exceptions while obeying the principle of least privilege and ensuring that unauthorized users do not acquire elevated privileges that they can abuse.

We now illustrate how our auto-delegation mechanism can be used to control positive exceptions. We assume that the access control mechanism enforces some authorization policy and that we can define a set of qualifications and a function $\lambda$ on the basis of that policy. Then we have a function $A u t h_{\text {adm }}$ that defines whether a request is authorized by the auto-delegation mechanism. The decision returned by this function is determined by the request, $\lambda$ and the set of available subjects. In other words, Auth $h_{\mathrm{adm}}$ is time-dependent and is determined by the set of available subjects (assuming that $\lambda$ is fixed).

In general, we would expect $S_{o}$ to contain those users that are authorized for $o$ and are "closest" or who have control over $o$ in some sense. In a discretionary model, for example, $S_{o}$ might only contain those subjects that are designated "owners" of $o$. In a multi-level model, $S_{o}$ might contain those subjects with the same security label as $o$.

The purpose of ADM in this context is to provide a policy-driven way for the PEP to generate positive exceptions. In the normal course of events, the PEP accepts any PDP decision to allow. However, the PEP may choose to refer a request that is not authorized to the ADM. If there are no other suitably authorized subjects available, the ADM will return an allow decision, which the PEP may use to implement a positive exception. This architecture is illustrated in Fig 4.

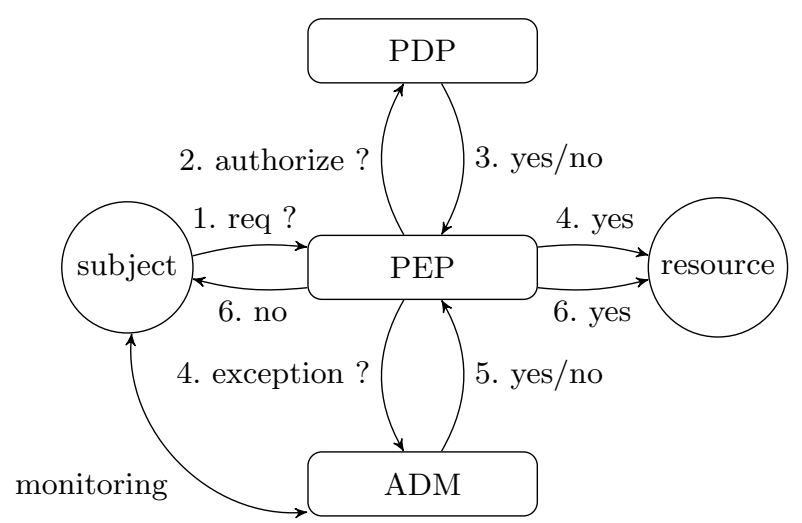

Fig. 4. Access Control System with ADM 
We expect that the circumstances under which the ADM will be invoked will vary from system to system. Here we consider a few of the possibilities.

1. We define $\lambda$ for a small number of objects and only invoke ADM for requests to those objects. It might be, for example, that certain objects in a missioncritical system must always be accessible to some subject.

2. We might, alternatively, only invoke the ADM when certain emergency conditions prevail. Under normal circumstances, for example, the PEP only considers the output of the PDP. However, when the context changes, the access control mechanism transfers to a new mode, in which the ADM may be used to override deny decisions from the PDP.

3. Finally, we might have a PEP that is "aware" of the availability of subjects and the impact that this might have on the accessibility of objects. In this case, the PEP might selectively and adaptively invoke the ADM. Such an approach might be appropriate in workflow management systems to ensure that certain workflow instances can be completed (by invoking the ADM for a particular business object in the critical path of the workflow instance). Workflow satisfiability $[8,27]$ and workflow resiliency $[27]$ have attracted interest in the research community in recent years, and ADM may provide a way of addressing some of the issues raised by this research.

\subsection{Standalone Policy}

Although our original intention was to design an exception mechanism that would allow auto-delegation to override an access control policy in exceptional circumstances, we believe that the ADM can also be used as a "pure" access control mechanism.

Consider the well known "owner-group-world" access control model used in Unix and its derivatives. This could be viewed as defining a set of qualifications, and every user belongs to (precisely) one of three qualifications. These qualifications are defined by the relationship that exists between the subject and object, the relationship being determined by the UIDs (user identifiers) and GIDs (group identifiers) that are associated with the subject and object and stored in the etc/passwd and etc/group files. Of course, each qualification is now associated with a permission mask, which is an extension of our current model for auto-delegation.

We believe that this approach could be extended to any application domain in which the authorization policy is in large part determined by the relationship that exists between subjects and objects. Two obvious examples, which have been extensively used as case studies in the access control literatures, spring to mind: higher education systems and healthcare systems.

In the first example, several different relationships might exist between a piece of student coursework (a protected resource) and subjects: the student herself is the "owner" of the resource; the teaching assistant assigned to the course may be the "marker" of the resource; the professor assigned to the course may be the "grader" of the resource; and so on. 
In the second example, an individual healthcare record may be the protected resource: the subject of the record is the "owner"; the personal physician is the next most privilege user with respect to this resource; then all doctors working on the patient's ward; then all nurses working on the patient's ward; then all doctors working in the hospital; and so on.

Now these relationships, in both cases, can be deduced from other information. The coursework can be identified with a particular course ID, each teaching assistant (TA) can be identified with a course ID, and so on. A healthcare management information system will define the patient-doctor relationships and the allocation of patients, nurses and doctors to wards, so all of the above relationships can (with appropriate system interaction, perhaps using web services, for example) be deduced at the time an access request is made. ${ }^{3}$

In this context, the auto-delegation mechanism must be extended to define the set of access rights that are associated with each qualification. In other words, we define an ADM, in which, for each protected resource we first define a set of named qualifications (owner, marker, grader, etc.); we then associate each subject with a qualification using a function defined by attributes retrieved from relevant sources; and finally we associate a permission mask with each qualification.

Space constraints do not permit a lengthy exposition of the use of ADM as an access control mechanism in its own right. This will be the subject of future research.

\section{Cryptographic Implementations of ADM}

Traditionally, an access control policy is enforced by a centralized mechanism (implemented in software, hardware, or both, and often called a reference monitor) that can evaluate every access request and permits only those requests that are authorized. The implementation of a reference monitor - commonly split into decision and enforcement modules (as shown in Fig. 1) - is well understood, and the implementation of the ADM alongside such technology appears to be straightforward. Another possibility is to use a cryptographic mechanism to enforce an authorization policy, and it is such techniques that are the focus of this section.

When using cryptographic access control, protected objects are encrypted and the authorization policy is enforced by distributing the appropriate decryption keys to authorized users (see $[2,9,10,18]$, for example). Typically, such a mechanism is used for protected objects to which only read access is controlled and the authorization policy is static (since changes to the policy would

\footnotetext{
${ }^{3}$ We note, in passing, that we remain unconvinced that RBAC is an appropriate solution for such applications. Most commonly, we see the use of parameterized roles [13], where we might have a personal physician role for each patient. But this, generally, results in an significant increase in the number of policy principals (roles in this case), something that RBAC, when it was introduced, was expressly seeking to avoid.
} 
generally require the revocation and redistribution of decryption keys and the re-encryption of protected objects). Generally, a central server acts as a repository for encrypted objects and provides them on request, the access control being realized by the ability of the end user to decrypt the object. However, this centralized approach has the disadvantage that the server may be a bottleneck and is not well-suited to distributed systems, where the mechanism becomes a single point of failure. In this section, we briefly consider the implementation of a centralized cryptographic mechanism for ADM before turning to the more interesting case of a decentralized mechanism.

\subsection{Centralized Cryptographic Implementation}

Following similar work in the literature $[9,10]$, we assume that every object $o$ is encrypted with some symmetric encryption key $\kappa(o)$, and that every subject $s$ shares a unique secret symmetric encryption key $\kappa(s)$ with the access control mechanism. ${ }^{4}$

Then the access control mechanism publishes $\mathrm{Enc}_{\kappa(s)}(\kappa(o))$ for every $s$ that is authorized to read $o$, where $\operatorname{Enc}_{k}(m)$ denotes the (symmetric) encryption of message $m$ with key $k$. Moreover, if every authorized $s$ is unavailable, the access control mechanism can publish Enc $_{\kappa\left(s^{\prime}\right)}(\kappa(o))$ for some appropriately qualified and available $s^{\prime}$. Alternatively, let $q \in Q$ denote the qualification level with the property that for all $q^{\prime}>q$, there is no available subject $s^{\prime}$ such that $\lambda\left(s^{\prime}\right)=q^{\prime}$. Then the access control mechanism can publish $\operatorname{Enc}_{\kappa(s)}(\kappa(o))$ for all $s$ such that $\lambda(s)=q$ and $s$ is available.

Of course, once additional information is published, the original authorization policy has been overridden and $o$ is now accessible to at least one user that was not previously able to access it. If $o$ is ever modified at some point in the future, then it would be appropriate to: select a new key $\kappa^{\prime}(o)$; re-encrypt $o$ with the new key; and (re)publish $E_{n c} c_{\kappa(s)}\left(\kappa^{\prime}(o)\right)$ for every authorized subject $s$.

\subsection{Decentralized Cryptographic Implementation}

The approach presented in the previous section relies on the existence of a central server that checks the availability of the subjects and distributes the key for a resource to the most qualified available subjects. The main drawback of this approach is that if this server is unavailable, then the policy can no longer be enforced. For instance, consider a military unit deployed "in theater", where the ADM is enforced within the unit. Either the server stays at the base, forcing the unit to have a permanent communication link with the base, which can be a problem if the deployment occurs far from the base or if the enemy can intercept or scramble the link, or the central server is deployed with the team, making it possible to be destroyed during an attack.

\footnotetext{
${ }^{4}$ An alternative assumption is that every subject is associated with a unique publicprivate key pair $(k(s), K(s))$ and that the access control mechanism possesses or can acquire an authenticated copy of the public key $k(s)$.
} 
One solution is to replicate the server within the team, making it more resilient to attacks, but at the same time creating more entities with the key, therefore increasing the probability of leaking it. We propose here an approach based on secret sharing: the key is shared in several pieces, one for each subject, such that all the pieces are needed to compute the key. Each subject initially owns all the shares except those of the subjects with a strictly greater qualification, and once a subject becomes unavailable, the corresponding share is broadcast to every other subject.

More formally, given a set of subjects $\mathcal{S}=\left\{s_{1}, \cdots, s_{n}\right\}$, an object $o$ is encrypted with a key $\kappa(o)$ such that $\kappa(o)=h\left(d_{0}, d_{1}, \cdots, d_{n}\right)$, where $h$ is a hash function, $d_{i}(1 \leq i \leq n)$ denotes a "share" associated with subject $s_{i}$, and $d_{0}$ is a special share, corresponding to no particular subject. At initialization time, for instance before the unit is deployed on the field, each subject is assigned the special share $d_{0}$ together with all the shares of the subjects that are not better qualified than her.

Since we do not assume the existence of any central server, the unavailability of subjects must be managed directly by the subjects themselves. For each subject $s_{i}$, we introduce a broadcast unit $b_{i}$, whose only role is to broadcast the share $d_{i}$ when $s_{i}$ becomes unavailable. In other words, the system is organized in a P2P network, where a peer is either a subject or a broadcast unit, and where each subject owns a copy of the encrypted resource and all the shares of the subjects that are not strictly more qualified. As long as a subject $s_{i}$ is available, she communicates with the corresponding broadcast unit $b_{i}$ not to broadcast the share $d_{i}$. If $s_{i}$ becomes unavailable, then she can no longer interact with $b_{i}$, which then broadcasts $d_{i}$ to every other subject.

For instance, consider an object $o$ and four subjects $s_{1}, s_{2}, s_{3}$ and $s_{4}$ such that $\lambda\left(s_{1}\right) \leqslant \lambda\left(s_{3}\right) \leqslant \lambda\left(s_{4}\right)$ and $\lambda\left(s_{2}\right) \leqslant \lambda\left(s_{3}\right) \leqslant \lambda\left(s_{4}\right)$. Then the key $\kappa(o)$ is defined to be $h\left(d_{0}, d_{1}, d_{2}, d_{3}, d_{4}\right)$. The shares owned by the subjects at system initialization are as follows:

$$
\begin{array}{l|l|l|l|l|l|}
s_{1}: & d_{0} & d_{1} & d_{2} & d_{3} & d_{4} \\
s_{2}: & d_{0} & d_{1} & d_{2} & d_{3} & d_{4} \\
s_{3}: & d_{0} & & & d_{3} & d_{4} \\
\hline
\end{array}
$$

The subjects $s_{1}$ and $s_{2}$ are able to decrypt $o$, since they have all the shares, while $s_{3}$ and $s_{4}$ are still missing some. If $s_{3}$ becomes unavailable, it will not change anything for $s_{1}$ and $s_{2}$, as they already have the share $d_{3}$, but then $s_{4}$ will only be missing $d_{1}$ and $d_{2}$. If $s_{1}$ becomes unavailable, then $d_{1}$ is broadcast to all the subjects, and therefore to $s_{4}$, but she still cannot compute $\kappa(o)$. Finally, if $s_{2}$ also becomes unavailable, then $s_{4}$ owns all the shares and can then access $o$.

Since the share $d_{0}$ is given to the subjects at initialization and does not correspond to any subject, this share is known by all the subjects but is never broadcast, making it impossible to get $\kappa(o)$ only by intercepting all the shares broadcast on the network. Moreover, each broadcast unit $b_{i}$ only knows the share 
$d_{i}$, so it can be replicated to be more resilient without risking the leakage of too much information.

The main advantage of this approach is that there is no single point of failure. However, this approach is only suitable for certain applications or in certain scenarios, because it relies on cryptographic access control, which requires the re-encryption of objects when a key is compromised. In the context of ADM, a key is revealed to unauthorized users when no authorized user is available, so a decentralized approach - which cannot co-ordinate the re-encryption of objects

- will only be appropriate if the re-encryption of objects is not required or if we may assume that a subject will remain unavailable once it becomes unavailable.

\section{Conclusion}

The main contribution of this paper is the definition of an auto-delegation mechanism (ADM), which associates each subject with a qualification for each object, and authorizes a subject to access an object if she is one of the most qualified available subjects for this object. Two concrete examples of the definition of qualifications are given, using the RBAC and the BLP models.

Related work The advantage of this approach compared to "break-the-glass" policies $[1,26]$ and contextual policies $[17]$ is that the original policy is not modified, and the ADM can be (optionally) enforced on top of any policy, assuming that a notion of qualification can be defined. In [5], a notion of distance function is introduced in order to find a mentor, that is a user that can either perform the desired operation or delegate the corresponding access rights, and such a notion could be used to build directly a hierarchy of qualifications.

The implementation of "break-the-glass" policies is addressed in [4], where the notion of emergency levels represents the extension of a policy with exceptions, and an implementation in XACML is given. Traditional delegation mechanisms [6] require a subject to explicitly delegate some rights, and have been extended in [16], to introduce a notion of capability attached to roles. However, these models assume that a user must be available to make the delegation, and hence must know the delegate, whereas we propose a notion of qualification defined directly by the administrator.

Future work As we said in Sec. 3.2, the use of ADM as an access control mechanism in its own right, in particular by associating different access modes with different qualifications, would be very appropriate within systems where the policy is determined by the relationship existing between entities. Another interesting possibility would be to use translation mechanisms between policies [14] to define the qualification. For instance, a hierarchy of roles can be automatically built from a lattice [23], therefore it would be interesting to compare the qualifications defined on such a hierarchy with the qualifications defined on the lattice.

In some contexts, when an object is accessed by a subject with a low qualification, even though this subject is the most qualified available, some parts 
might need to be filtered out [7,22]. For instance, if the medical record of a patient should be accessed by someone else than the official physician in order to perform an emergency treatment, the sexual history can be erased from the record.

Moreover, the ADM may provide some leads in the research concerning workflow satisfiability $[8,27]$ and workflow resiliency [27], as the notion of availability is central in these research topics.

Acknowledgements The authors thank Pau-Chen Cheng and Pooya Farshim for enlightening discussions on this subject. We would also like to express our appreciation to the reviewers, whose remarks helped to improve the paper significantly.

This research was sponsored in part by the US Army Research Laboratory and the UK Ministry of Defence and was accomplished under Agreement Number W911NF-06-3-0001. The views and conclusions contained in this document are those of the authors and should not be interpreted as representing the official policies, either expressed or implied, of the US Army Research Laboratory, the US Government, the UK Ministry of Defense, or the UK Government. The US and UK Governments are authorized to reproduce and distribute reprints for Government purposes notwithstanding any copyright notation hereon.

\section{References}

1. C.A. Ardagna, S. Capitani Di Vimercati, T. Grandison, S. Jajodia, and P. Samarati. Regulating exceptions in healthcare using policy spaces. In Proceeedings of the 22nd Annual IFIP WG 11.3 Working Conference on Data and Applications Security, pages 254-267, Berlin, Heidelberg, 2008. Springer-Verlag.

2. M. Blaze. A cryptographic file system for UNIX. In 1st ACM Conference on Computer and Communications Security, pages 9-16, 1993.

3. D. F. C. Brewer and M. J. Nash. The Chinese Wall Security Policy. In Proceedings of the IEEE Symposium on Security and Privacy, pages 329-339, May 1989.

4. A. D. Brucker and H. Petritsch. Extending access control models with breakglass. In SACMAT '09: Proceedings of the 14th ACM symposium on Access control models and technologies, pages 197-206, New York, NY, USA, 2009. ACM.

5. A. D. Brucker, H. Petritsch, and A. Schaad. Delegation assistance. Policies for Distributed Systems and Networks, IEEE International Workshop on, 0:84-91, 2009.

6. A. Chander, J.C. Mitchell, and D. Dean. A state-transition model of trust management and access control. In Proceedings of the 14th IEEE Computer Security Foundations Workshop, pages 27-43. IEEE Computer Society Press, 2001.

7. J. Crampton. Applying hierarchical and role-based access control to XML documents. In Proceedings of 2004 ACM Workshop on Secure Web Services, pages 41-50, 2004

8. J. Crampton. A reference monitor for workflow systems with constrained task execution. In Proceedings of the 10th ACM Symposium on Access Control Models and Technologies, pages 38-47, 2005.

9. J. Crampton, K.M. Martin, and P. Wild. On key assignment for hierarchical access control. In Proceedings of 19th Computer Security Foundations Workshop, pages 98-111, 2006. 
10. S. De Capitani di Vimercati, S. Foresti, S. Jajodia, S. Paraboschi, and P. Samarati. Encryption policies for regulating access to outsourced data. ACM Transactions on Database Systems, 35(2), 2010.

11. D.E. Denning. A Lattice Model of Secure Information Flow. Communications of the ACM, 19(5):236-243, 1976.

12. D. F. Ferraiolo and D. R. Kuhn. Role-based access control. In Proceedings of the 15th National Computer Security Conference, pages 554-563, 1992.

13. L. Giuri and P. Iglio. Role templates for content-based access control. In Proceedings of Second ACM Workshop on Role-Based Access Control, pages 153-159, 1997.

14. L. Habib, M. Jaume, and C. Morisset. Formal definition and comparison of access control models. Journal of Information Assurance and Security, 4:372-381, 2009.

15. M. A. Harrison, W. L. Ruzzo, and J. D. Ullman. Protection in operating systems. Communications of the ACM, 19(8):461-471, 1976.

16. K. Hasebe, M. Mabuchi, and A. Matsushita. Capability-based delegation model in RBAC. In SACMAT '10: Proceeding of the 15th ACM Symposium on Access Control Models and Technologies, pages 109-118, New York, NY, USA, 2010. ACM.

17. A. Abou El Kalam, R. El Baida, P. Balbiani, S. Benferhat, F. Cuppens, Y. Deswarte, A. Miège, C. Saurel, and G. Trouessin. Organization based access control. In Proceedings of Policies for Distributed Systems and Networks, pages 120 - 131, Como, Italy, June 2003.

18. M. Kallahalla, E. Riedel, R. Swaminathan, Q. Wang, and K. Fu. Plutus: Scalable secure file sharing on untrusted storage. In Proceedings of the FAST' 03 Conference on File and Storage Technologies, pages 29-42, 2003.

19. B. Lampson. Protection. In Proceedings of the 5th Annual Princeton Conference on Information Sciences and Systems, pages 437-443, Princeton University, 1971.

20. L.J. LaPadula and D.E. Bell. Secure Computer Systems: A Mathematical Model. Journal of Computer Security, 4:239-263, 1996.

21. I. Mavridis and G. Pangalos. eMEDAC: Role-based access control supporting discretionary and mandatory features. In Proceedings of 13 th IFIP WG 11.3 Working Conference on Database Security, pages 63 - 78, 1999.

22. G. Miklau and D. Suciu. Controlling access to published data using cryptography. In Proceedings of 29th International Conference on Very Large Data Bases (VLDB 2003), pages 898-909, 2003.

23. S.L. Osborn, R.S. Sandhu, and Q. Munawer. Configuring role-based access control to enforce mandatory and discretionary access control policies. ACM Transactions on Information and System Security, 3(2):85-106, 2000.

24. J. Reid, I. Cheong, M. Henricksen, and J. Smith. A novel use of RBAC to protect privacy in distributed health care information systems. In Proceedings of the 8th Australasian Conference on Information Security and Privacy, pages 403-415, 2003.

25. R. S. Sandhu, E. J. Coyne, H. L. Feinstein, and C. E. Youman. Role-based access control models. IEEE Computer, 29(2):38-47, 1996.

26. J. Wainer, P. Barthelmess, and A. Kumar. W-RBAC - a workflow security model incorporating controlled overriding of constraints. International Journal of Cooperative Information Systems, 12:455-485, 2003.

27. Q. Wang and N. Li. Satisfiability and resiliency in workflow systems. In Proceedings of 12th European Symposium on Research in Computer Security, pages 90-105, 2007. 